\title{
Signal-to-Noise Ratio of Intensity Interferometry Experiments with Highly Asymmetric X-ray Sources
}

\author{
Y. P. Feng, I. McNulty, Z. Xu, and E. Gluskin \\ Experimental Facilities Division, Argonne National Laboratory, \\ Argonne, IL 60439
}

We discuss the signal-to-noise ratio of an intensity interferometry experiment for a highly asymmetric $\mathrm{x}$-ray source using different aperture shapes in front of the photodetectors. It is argued that, under ideal conditions using noiseless detectors and electronics, the use of slitshaped apertures, whose widths are smaller but whose lengths are much greater than the transverse coherence widths of the beam in the corresponding directions, provides no signal-tonoise advantage over the use of pinhole apertures equal to or smaller than the coherence area. As with pinholes, the signal-to-noise ratio is determined solely by the count degeneracy parameter and the degree of coherence of the beam. This contrasts with the signal-to-noise ratio enhancement achievable using slit-shaped apertures with an asymmetric source in a Young's experiment. 


\section{DISCLAIMER}

This report was prepared as an account of work sponsored by an agency of the United States Government. Neither the United States Government nor any agency thereof, nor any of their employees, make any warranty, express or implied, or assumes any legal liability or responsibility for the accuracy, completeness, or usefulness of any information, apparatus, product, or process disclosed, or represents that its use would not infringe privately owned rights. Reference herein to any specific commercial product, process, or service by trade name, trademark, manufacturer, or otherwise does not necessarily constitute or imply its endorsement, recommendation, or favoring by the United States Government or any agency thereof. The views and opinions of authors expressed herein do not necessarily state or reflect those of the United States Government or any agency thereof. 


\section{DISClaAMIER}

Portions of this document may be illegible in electronic image products. Images are produced from the best available original document. 


\section{INTRODUCTION}

The prospects for an x-ray Hanbury Brown and Twiss (HBT) intensity interferometry experiment using synchrotron sources have been considered for some time.[1-4] The success of such an experiment hinges largely on the spectral brightness of the source, and thus calls for the use of high brightness $\mathrm{x}$-ray sources, such as undulators. An important characteristic of undulator sources is that they are much larger in the horizontal than in the vertical extent.[5] According to the Van Cittert-Zernike theorem, ${ }^{[6]}$ the transverse coherence function of the beam emitted by a spatially incoherent source, at a plane of observation normal to the beam, is determined entirely by the intensity distribution of the source. Undulator sources are essentially incoherent. Consequently, the coherence width of an undulator beam is usually much greater in the vertical than in the horizontal direction.

In order to measure the vertical coherence profile of the $x$-ray beam produced by an undulator, we recently constructed a soft $x$-ray intensity interferometer and installed it on the X13A undulator beamline at the National Synchrotron Light Source (NSLS). ${ }^{77-9]}$ To take advantage of the asymmetric beam profile, we proposed the use of slit-shaped apertures whose widths are smaller than the vertical coherence width of the beam, but whose lengths are equal to the horizontal beam size, which is much greater than the horizontal coherence width. At the time, we concluded that a larger signal-to-noise ratio (SNR) can be achieved by using this geometry. ${ }^{[7]}$ We have since realized that, under ideal experimental conditions (for example, noiseless electronics), the use of slit-shaped apertures will not enhance the SNR over that which would pertain using pinholes. In this report we present the arguments that lead to this new conclusion. We will also contrast the independence of the SNR on the slit length for a HBT experiment with the SNR advantage of using slit apertures in a Young's experiment.

\section{SOURCE AND COUNT DEGENERACY PARAMETERS}

Our intensity interferometer is an $\mathrm{x}$-ray version of the original Hanbury Brown and Twiss 
'experiment with visible light, $\{10]$ in which the correlation of the intensity fluctuations at two spatially separated points (defined by two detector apertures) in a partially coherent beam are measured and used to determine the modulus of the complex degree of coherence between these two points. The figure of merit for a HBT experiment is the source degeneracy parameter $\delta$, which is the number of photons emitted by the source per spatially and temporally coherent mode. $\delta$ depends on fundamental properties of the source and is given by

$$
\delta=B \lambda^{3} / 4 c
$$

where $B$ is the spectral brightness of the source defined as the number of photons emitted per unit time, source area, solid angle, and bandwidth, $\lambda$ is the wavelength, and $c$ is the speed of light.[6] For synchrotron $\mathrm{x}$-ray sources, because of their pulsed time structure, one must distinguish the time-averaged brightness from the peak brightness, which is greater and is inversely proportional to the pulse duty-cycle. The pulsed time structure and larger peak brightness may entail certain SNR advantages and disadvantages in a real x-ray HBT experiment.[11] However, in order to simplify the discussion below, we assume that the $\mathrm{x}$-ray source is continuous.

Of practical interest here is the count degeneracy parameter, ${ }^{[10]}$ which accounts for the relative sizes of the detector apertures with respect to the beam coherence area. In an earlier paper, ${ }^{[7]}$ we proposed that when slit-shaped detector apertures accept the entire horizontal extent of an undulator beam but only accept a fraction of the coherent portion in the vertical direction, an effective count degeneracy parameter,

$$
\begin{aligned}
\delta_{y} & =\iint_{\Sigma_{z} \Sigma_{x^{\prime}}} \delta \frac{d x d x^{\prime}}{(\lambda / 2)} \equiv \delta \frac{\Sigma_{x} \Sigma_{x^{\prime}}}{(\lambda / 2)}, \\
& =M_{x} \delta
\end{aligned}
$$

applies, where $\Sigma_{x}$ and $\Sigma_{x^{\prime}}$ are the horizontal source size and angular divergence, respectively, and $M_{x}=\Sigma_{x} \Sigma_{x^{\prime}} /(\lambda / 2)$ is the number of horizontal spatial modes accepted by the slits. In the previous experiment we performed on the X13A beamline at NSLS, $M_{x}$ was of the order of $10^{2}$ 
for the $32 \AA x$-rays produced by the undulator. ${ }^{[8]}$ The resulting $\delta$ was presumed to enhance the SNR for a given correlation integration time $T$, or conversely, to reduce the time required to achieve a predetermined SNR, because $T$ is inversely proportional to $\delta_{y}{ }^{\left[{ }^{[8]}\right.}$

However, Eqn (2) is incorrect because the count degeneracy can never exceed the source degeneracy if the phase space of the beam is to be conserved in the absence of dissipation. Instead, we consider the count degeneracy parameter of Goodman ${ }^{[12]}$

$$
\delta_{c}=\bar{K} / M
$$

where $\bar{K}=I \eta A \tau_{r}$ is the average number of photon counts detected in an interval $\tau_{r}$ by a photodetector illuminated with irradiance $I$, with area $A$ and quantum efficiency $\eta$, and $M=M_{s} M_{t}$ is the number of spatial and temporal modes accepted by the photodetector in $\tau_{r}$. By comparison to the source degeneracy, $\delta_{c}$ is the number of photons that are detected per coherent mode. If we restrict ourselves to the case of photon detection by primary photoelectric effect, $\eta$ is always less than unity and $\delta_{c}$ is always less than or equal to $\delta$. For an experiment with an x-ray beam of coherence area $A_{c}>A$, we have $M_{s}=1$, and $M_{t}=\tau_{r} / \tau_{c}$, where $\tau_{c}=\lambda 2 / c \Delta \lambda$ is the coherence time of the beam. Eqn. (3) gives

$$
\delta_{c}=\frac{I \eta A \tau_{r}}{M_{t}}=\eta \delta \frac{A}{A_{c}}
$$

with $\delta \approx I A_{c} \tau_{c}$ being just the source degeneracy.[11] The exact proportionality depends on the spatial and spectral shapes of the source and the coherence criteria used. Eqn. (4) now accounts correctly for the relative sizes of the apertures with respect to the coherence area. One can increase $\delta_{c}$ up to the limiting value of $\delta$ by increasing the area of the apertures.

For a highly asymmetric source, such as an undulator, Eqn. (4) must be modified accordingly. The source asymmetry (the ratio of the horizontal to vertical extent is about 40 in our case) allows separation of variables such that the beam coherence function can be taken as 
'the product of two independent functions, each depending only on one of the two orthogonal coordinates $x$ and $y$ in the plane perpendicular to the beam. For slit-shaped apertures of lengths $L_{x}$ and $L_{y}$ such that $L_{y}<L_{y c}$ and $L_{x}>L_{x c}$, where $L_{x c}$ and $L_{y c}$ are the spatial coherence widths of the beam at the location of the apertures in the horizontal and vertical directions, respectively, we have $M_{s}=M_{x} M_{y}=L_{x} / L_{x c}$, with $M_{y}=1$, and

$$
\delta_{c}=\frac{\operatorname{I\eta } L_{x} L_{y} \tau_{r}}{M_{s} M_{t}}=\eta \delta \frac{L_{y}}{L_{y c}},
$$

which is independent of the horizontal slit length $L_{x}$ and is always less than $\delta$.

On account of Eqn (5), we argue that the SNR for a HBT experiment is independent of the horizontal lengths of the slits provided the slits are longer than the horizontal coherence width. The signal, however, does increase linearly with the slit length. Hence under practical conditions, the slit geometry could offer certain SNR advantages over the pinhole geometry because it could allow the signal to dominate over extrinsic noise from detectors and electronics in a real experiment. For the sake of clarity, we first reiterate the SNR calculation by Goodman[12] for pinhole apertures, but with asymmetric sources. We then discuss the case for slit apertures with asymmetric sources applicable to our x-ray HBT interferometer.

\section{SNR IN INTENSITY INTERFEROMETRY}

\section{(i) Pinhole Apertures}

Although the experiment mentioned above was performed in the so-called "currentmode,"[8] we consider a "counting-mode" experiment for this discussion. We emphasize that these two approaches should give equivalent results. It is also assumed that the undulator source exhibits thermal-like statistics and that the radiation emitted is linearly polarized and crossspectrally pure. ${ }^{[12]}$ The intensity interferometer consists of two photodetectors and an electronic correlator. The acceptances of the photodetectors are defined by two pinholes apertures as 
'shown schematically in Fig. 1(a), and it is assumed that the diameter of the pinholes is no larger than $L_{x o}$ the smaller of the two coherence widths of the beam in the observation plane.

In the "counting-mode," photons passing through the two apertures 1 and 2 are detected and counted independently. The number of counts is recorded over a finite time interval $\tau_{r}$, resulting in two signals $K_{1}$ and $K_{2}$. We assume that $\tau_{r} \gg \tau_{c}$. The counting is repeated $m$ times with $m$ being a very large number. Simultaneously, the two signals for each $\tau_{r}$ are multiplied by the electronic correlator, and their products are summed over a time $T=m \tau_{r}$. The output of the correlator, which measures the coincidences of $K_{1}$ and $K_{2}$, has been shown to be[11]

$$
\sum_{m} K_{1} K_{2}=m \bar{K}_{1} \bar{K}_{2}\left[1+\frac{\tau_{c}}{\tau_{r}}\left|\mu_{12}(x) \mu_{12}(y)\right|^{2}\right] \text {, }
$$

where $\bar{K}_{1}$ and $\bar{K}_{2}$ are the average counts per interval $\tau_{r}$ over $m$ intervals, and $\mu_{12}(x)$ and $\mu_{12}(y)$ are the complex degrees of coherence between points 1 and 2 in the $x$ and $y$ directions. The first term in Eqn. (6) corresponds to the random coincidence rate, whereas the second term measures correlation between the fluctuations $\Delta K_{1}=K_{1}-\bar{K}_{1}$ and $\Delta K_{2}=K_{2}-\bar{K}_{2}$ in the two signals and represents the true coincidence rate due to photon bunching or classical intensity fluctuations in a partially coherent beam produced by a thermal-like source.[13] Assuming the fields at points 1 and 2 are horizontally coherent such that $\left|\mu_{12}(x)\right|=1$, the average correlation signal per counting interval $\tau_{r}$, due to true coincidences, becomes,

$$
S_{1}=\frac{1}{m} \sum_{m} \Delta K_{1} \Delta K_{2}=\bar{K}_{1} \bar{K}_{2}\left[\frac{\left|\mu_{12}(y)\right|^{2}}{M_{t}}\right] \text {, }
$$

where $M_{t}=\tau_{r} / \tau_{c}$ is just the number of temporally coherent modes included in each counting interval. Note that $S_{1}$ vanishes in the absence of spatial coherence, and also that a longer counting interval $\tau_{r}$ (i.e., a worse time resolution) tends to reduce the true coincidence rate as seen by the correlator. 
The noise in the correlation signal has two components, one arising from fluctuations in the true coincidence rate due to photon bunching and the other from those in the random coincidence rate due to the photon counting statistics (shot noise). In our case in which the source degeneracy parameter $\delta \ll 1$, the latter dominates the former. Therefore $\Delta K_{1}$ and $\Delta K_{2}$ can be treated as independent Poisson variates and their correlation neglected in evaluating the average noise $N_{1}$ per counting interval, which becomes[12]

$$
\begin{aligned}
N_{1} & =\sqrt{\overline{\left(\Delta K_{1} \Delta K_{2}\right)^{2}}-\left(\overline{\Delta K_{1} \Delta K_{2}}\right)^{2}} \\
& \cong \sqrt{\Delta K_{1}^{2} \overline{\Delta K_{2}^{2}}}=\sqrt{\bar{K}_{1} \bar{K}_{2}} .
\end{aligned}
$$

It is important to note that $N_{1}$ in Eqn. (5) is independent of the pinhole locations, which may be inside two different coherence areas of the beam. For a much brighter source where the noise associated with the photon bunching dominates the shot noise, Eqn. (5) would no longer be valid, and the noise would also depend on the correlation between the interfering signals. In the present case, the SNR for one counting interval is given by

$$
S N R_{1}=\frac{\bar{K}}{M_{t}}\left|\mu_{12}(y)\right|^{2}=\delta_{c}\left|\mu_{12}(y)\right|^{2},
$$

where $\bar{K}$ is the average count per interval assuming $\bar{K}_{1}=\bar{K}_{1}=\bar{K}$, and the count degeneracy parameter $\delta_{c}$ is defined in Eqn. (4). Since $\tau_{c} \ll<\tau_{r}$, the fluctuations in one counting interval can be considered to be uncorrelated from those in the next, and the SNR for $m$ intervals is then proportional to the square root of $m$, i.e.,

$$
S N R_{m}=\sqrt{m} \frac{\bar{K}}{M_{i}}\left|\mu_{12}(y)\right|^{2}=\sqrt{m} \delta_{c}\left|\mu_{12}(y)\right|^{2} .
$$

To increase the SNR, one can increase $\delta_{c}$ by enlarging the area of the pinholes up to the coherence area, but only at the expense of spatial resolution of the measurement. 


\section{(b) Slit-Shaped A pertures:}

One way to increase the correlation signal is to increase $\bar{K}_{1}$ and $\bar{K}_{2}$ in Eqn. (7). This can be done by increasing the aperture size using slit-shaped apertures whose longer dimensions are aligned along the $x$-axis, as depicted in Fig. 1(b). We assume that the widths of the slits $L_{y 1}$ and $L_{y 2}$ are smaller than $L_{y o}$ the coherence width of the beam in the $y$-direction, but the lengths of the slits $L_{x 1}$ and $L_{x 2}$ are much greater than $L_{x c}$, the coherence width in the $x$-direction. For simplicity, we further assume that $L_{y 1}=L_{y 2}=L_{y}, L_{x 1}=L_{x 2}=L_{x}$, and $M_{x}=L_{x} / L_{x c} \gg 1$. We divide both slits into $M_{x}$ imaginary subcells with each subcell (shaded area) traversing exactly one coherence width $L_{x c}$ as depicted in Fig. 1(b). Hence, there are $M_{x}$ modes enclosed by the slits as opposed to one in the pinhole geometry.

Eqns. (6) through (10) must now be generalized for $L_{x}>L_{x c}$, i.e., when more than one spatial mode is accepted by the detectors. We still consider $m$ counting intervals $\tau_{r}$ for a total time of $T$. The correlator signal per counting interval becomes:

$$
\begin{aligned}
S_{1} & =\frac{1}{m} \sum_{m} \Delta K_{1} \Delta K_{2}=\left(M_{x} \bar{K}\right)\left(M_{x} \bar{K}\right)\left[\frac{\left|\mu_{12}(y)\right|^{2}}{M_{x} M_{t}}\right] \\
& =M_{x} \bar{K}^{2}\left[\frac{\left|\mu_{12}(y)\right|^{2}}{M_{t}}\right]
\end{aligned},
$$

where $\bar{K}$ is the average count arising from each subcell, $M_{x} \bar{K}=\left.\bar{K}\right|_{L_{x}}$ is the average total count from one slit. The appearance of $M_{x}$ in the denominator of Eqn. (7') is due to the acceptance of more than one spatial mode by the detectors. When $M_{x} \rightarrow 1$ as in Fig. 1(a), Eqn. (7') is reduced to Eqn. (7) as expected. From Eqn. (7'), we see that the inclusion of $M_{x}$ modes increases the detector signals but also results in a loss of spatial resolution and tends to reduce the true coincidence rate resolved by the correlator in the same way as $M_{i}$. Consequently, in comparison with Eqn. (7), the correlation signal increases only linearly with $M_{x}$ or the slit length $L_{x}$. 
The intriguing question to ask here is whether the noise level remains the same as that for the pinhole geometry. In our previous analysis, ${ }^{[8]}$ the noise level was assumed to remain unchanged. If we again consider only the pure shot noise and ignore the noise in the true coincidences, the average noise per counting interval will be

$$
N_{1}=\sqrt{\left(M_{x} \bar{K}\right)\left(M_{x} \bar{K}\right)}=M_{x} \bar{K} \text {, }
$$

increasing linearly with $M_{x}$ or the slit length $L_{x}$. Therefore the SNR per counting interval for the slit geometry is

$$
S N R_{1}^{s l i t}=\frac{\left.\bar{X}\right|_{L_{x}}}{M_{x} M_{t}}\left|\mu_{12}(y)\right|^{2}=\delta_{c}\left|\mu_{12}(y)\right|^{2},
$$

where we have again made use of the count degeneracy of equations (3) to (5), but this time with $\delta_{c}=\eta \delta L_{y} / L_{y c}$ as defined in Eqn. (5) to account for the coherence widths and aperture sizes. As in Eqn. (10), the SNR of the slit geometry for $m$ counting intervals is proportional to the square-root of $m$, i.e.,

$$
\left.S N R\right|_{m} ^{s l i s}=\sqrt{m} \delta_{c}\left|\mu_{12}(y)\right|^{2}
$$

Clearly, the SNR is independent of the slit length $L_{x}$. The count degeneracies using pinholes or slits are identical if the pinhole area is $A=L_{x c} L_{y}$ and the coherence area of the beam is $A_{c}=$ $L_{x c} L_{y c}$. One can increase the SNR by increasing the width $L_{y}$ of the slits but again at the expense of spatial resolution.

To help appreciate this conclusion, one can imagine the slit experiment as a combination of many simultaneous "pinhole" experiments. As depicted in Fig. 2(a), there are now $M_{x}$ separate pairs of mutually coherent subcells or "pinholes" across the slits, with each subcell occupying an equal area $L_{x c} L_{y}$. The total correlation signal from the two slits is given by the incoherent sum of the individual correlation signals arising from all mutually coherent subcell 
'pairs. From Eqn. (7), we have:

$$
S_{s l i t}=S_{1}+S_{2}+\ldots+S_{i}+\ldots+S_{M_{x}}=M_{x} \bar{K}^{2}\left[\frac{\left|\mu_{12}(y)\right|^{2}}{M_{t}}\right] \text {, }
$$

where $S_{i}=\overline{\Delta K_{1 i} \Delta K_{2 i}}$ is the correlation signal due to the signal $K_{1 i}$ from the $i$ th subcell in the upper slit and the signal $K_{2 i}$ from the $i$ th subcell in the lower slit, and we assume that $\bar{K}_{1 i}=\bar{K}_{2 i}=\bar{K}$. The correlation signal produced by the two subcell signals $K_{1 i}$ and $\mathrm{K}_{2 j}$, for $i \neq j$ such that these two subcells do not reside within a single coherence area, is identically zero because of the lack of correlation between their fluctuations.

The noise in the correlation signal is calculated differently. The noise in the random coincidence rate due to $K_{1 i}$ and $K_{2 j}$ is nonzero for $i \neq j$, and is independent of the subcell locations in the slits, i.e.,

$$
N_{i j}=\sqrt{\bar{K}_{1 i} \bar{K}_{2 j}}=\bar{K}
$$

as in Eqn. (8). Because the noise arising from each individual pair of subcells is statistically uncorrelated to that from another, the total noise-squared is the sum of the noise-squared of all possible subcell combinations as illustrated in Fig. 2(b). The total noise-squared of the slit geometry thus becomes

$$
N_{s l i t}^{2}=\sum_{i}^{M x} \sum_{j}^{M x} N_{i j}^{2}=M_{x}^{2} \bar{K}^{2}
$$

consistent with Eqn. (8'). By Eqns. (11) and (13), the SNR of the slit geometry for one counting interval is again independent of the mode number $M_{x}$. The effect of the source and aperture shapes on the SNR has already been discussed by Hanbury Brown and Twiss, ${ }^{[14]}$ although they made no specific reference to a highly asymmetric source as in the present case. 


\section{SNR IN YOUNG'S EXPERIMENTS}

The essential difference between a Young's double-slit experiment and a HBT experiment is how the input signals are processed. The two interfering signals, i.e., the electric field amplitudes in the case of a Young's experiment, are combined before detection; whereas in the case of a HBT experiment, the signals (field intensities) are first detected and then combined electronically. Let us first consider the pinhole geometry shown in Fig. 1(a) with quasimonochromatic illumination. The average number of photocounts measured in an interval $\tau_{r}$ at a point $P$ in a plane of observation located at some distance behind the pinholes, is given by

$$
\begin{aligned}
\bar{K}(P) & =\bar{K}_{1}+\bar{K}_{2}+2 \sqrt{\bar{K}_{1} \bar{K}_{2}}\left|\mu_{12}(y)\right| \cos (k \Delta L) \\
& =\left(\bar{K}_{1}+\bar{K}_{2}\right)(1+V \cos (k \Delta L))
\end{aligned}
$$

where $\bar{K}_{1}$ and $\bar{K}_{2}$ are the average counts that would be measured at $P$ when one or the other pinhole is illuminated, $V=2 \sqrt{\bar{K}_{1} \bar{K}_{2}} / \mu_{12} / /\left(\bar{K}_{1}+\bar{K}_{2}\right)$ is the visibility of the fringes that are observed, $\Delta L$ is the path difference between $\mathrm{x}$-rays passing through the upper and lower pinholes that reach $P$, and $k=2 \pi / \lambda$.

For simplicity we assume that $\bar{K}_{1}=\bar{K}_{2}=\bar{K}$. The mean signal in the Young's experiment is identified with the amplitude of the interference term $\sqrt{2}\left|\mu_{12}\right| \bar{K}$, whereas the rms noise arises from the background term $2 \bar{K}$. Because $\bar{K}$ is a Poisson variate, the noise due to $\bar{K}$ is just $\sqrt{\bar{K}}$ and the SNR is

$$
S N R=\frac{\sqrt{2}\left|\mu_{12}\right| \bar{K}}{\sqrt{2 \bar{K}}}=\left|\mu_{12}\right| \sqrt{\delta_{c}} \sqrt{\frac{\tau_{r}}{\tau_{c}}}
$$

where $\delta_{c}=\bar{K} / M_{t}$ as before. In comparison with Eqn. (9), we see that the SNRs of the Young's and HBT experiments are distinguished by their dependence on the count degeneracy. It is also apparent that the SNR is proportional to $\sqrt{\bar{K}}$ in the Young's case as opposed to $\bar{K}$ in the HBT experiment. 
Based on Eqn. (15), a greater SNR can be obtained by increasing the field intensity at $P$, such as using the slit geometry as we consider now. The lengths of the slits $L_{1}$ and $L_{2}$ are assumed to be much greater than the horizontal coherence width $L_{x c}$ of the beam. Both slits are divided into $M_{x}$ imaginary subcells with each subcell traversing exactly one coherence width $L_{x c}$, as depicted in Fig. 1(b), where $M_{x}=L_{x} / L_{x c}>1$ and $L_{\mathrm{x}}=L_{1}=L_{2}$ as previously assumed. The number of counts measured at $P$ becomes

$$
\bar{K}(P)=2 M_{x} \bar{K}\left(1+\left|\mu_{12}\right| \cos (k \Delta L)\right),
$$

where $\bar{K}$ is the average count per interval $\tau_{r}$ produced by one coherent subcell either in the upper or lower slit, and $M_{x} \bar{K}$ is the average total count per interval $\tau_{r}$ from one slit. Following similar arguments in deriving Eqn. (15), the SNR for the slit geometry is

$$
S N R=\frac{\sqrt{2}\left|\mu_{12}\right| M_{x} \bar{K}}{\sqrt{2 M_{x} \bar{K}}}=\left|\mu_{12}\right| \sqrt{M_{x}} \sqrt{\delta_{c}} \sqrt{\frac{\tau_{r}}{\tau_{c}}},
$$

which depends on the length of the slits or the number of horizontally coherent modes. This is not surprising because the Young's signal for the slit geometry can be considered as the incoherent sum of the signals arising from $M_{x}$ pairs of mutually coherent subcells or "pinholes", whereas the total noise is that of $2 M_{x}$ independent Poisson variates; which depends on the squareroot of $M_{x}$. It is therefore beneficial to use slits instead of pinholes when performing a Young's experiment.

\section{DISCUSSION}

For a longer counting time $T=m \tau_{r}$, we find, from equations (15) and (15'), that the SNR $\propto \sqrt{m}$, which is identical to the time dependence of the HBT experiment. But, because the summation is done after the signal processing in the Young's experiment, the SNR is larger when using slits than that due to the pre-processing averaging of the signal in a HBT experiment. 
The reason for the independence of the SNR on the slit length in the HBT experiment is that the contribution to the signal from each "pinhole" pair is not independently processed. Consequently, the correlator sees the total noise in addition to signal from all "pinhole" pairs at once. The advantage of having a stronger signal is canceled out by the disadvantage of including more shot noise. The noise is greater due to contributions from all "pinhole" pairs, including those that are mutually coherent as well as incoherent. In contrast, the noise in Young's experiment is less because there is no contribution from mutually incoherent "pinhole" pairs.

However, there is a way to enhance the SNR of a HBT experiment using one-dimensional array detectors whose one spatial dimension is greater than the coherence width in the corresponding direction. Consider the scheme shown in Fig. 3, which is similar to that considered by Howells. [2] For simplicity, we assume that the size of each detection element of the detector matches the coherence width $L_{x c}$ and that there are $M_{x}$ of these elements in each detector. The detection electronics are configured such that the signals arising from each pair of elements located within a single coherence area are correlated independently and then averaged, after correlating them. Compared to equations $(10)$ and $\left(10^{\prime}\right)$, the SNR is enhanced by the square-root of the number of horizontal modes $M_{x}$, i.e.,

$$
\left.S N R\right|_{m}=\sqrt{M_{x}} \sqrt{m} \delta_{c}\left|\mu_{12}(y)\right|^{2}
$$

Correspondingly, the reduction of the measurement time can be substantial if $M_{x}$ is large. However, the above scheme may be difficult to implement experimentally because $M_{x}$ sets of amplification electronics and correlators would be needed.

The above analysis may be generalized to measurements involving a higher-order coherence function of degree $n$. In general, we expect the signal-to-noise ratio of the geometry in Fig. 1(b) to vary as $M_{x}^{1-n / 4}$, where $M_{x}$ is the number of horizontal modes accepted by the slits. We see that the signal in the slit geometry is always proportional to the length of the slits; whereas the noise varies as $M_{x}^{n / 4}$. For example, in an experiment designed to be sensitive to the 
-sixth-order degree of coherence, use of the slit configuration in Fig. 1(b) will reduce the SNR that one would achieve using the pinhole configuration in Fig. 1(a). In this case, the SNR for the slit geometry would vary as $1 / \sqrt{M_{x}}$.

Finally, we reestimate the minimum time $T_{\min }$ required to achieve a predetermined SNR for a HBT experiment with a synchrotron source using either the pinhole or slits geometry. For simplicity, we assume the source is continuous. By Eqn. $\left(10^{\prime}\right)$,

$$
T_{\min }=\frac{(S N R)^{2}}{D Q E} \frac{\tau_{r}}{\left(\eta_{b} \delta f_{y}\right)^{2}\left|\mu_{12}(y)\right|^{4}}
$$

where DQE is the detective quantum efficiency of the detector, $\eta_{b}$ is the beamline efficiency, and $f_{y}=L_{y} / L_{y c}$ is the coherent fraction accepted by the detector apertures. The time-averaged spectral brightness of the X13A undulator is approximately $1.0 \times 10^{17}$ photons $/\left(\mathrm{s}^{\bullet} \mathrm{mm}^{2} \cdot \mathrm{mrad}^{2} \cdot 0.1 \% \mathrm{BW}\right)$ at a wavelength of $3.2 \mathrm{~nm}$, amounting to a source degeneracy $\delta$ of $2.7 \times 10^{-3}$. If one were to measure values of $\left|\mu_{12}(y)\right|$ as small as 0.2 with a SNR of 3 , using $\eta_{b}=10 \%, \tau_{r}=3 \mathrm{~ns}, f_{y}=10 \%$, and DQE of $10 \%$, the required time is $T_{\min }=2.3 \times 10^{5} \mathrm{sec}$ or 64 hours, which is impractical for a synchrotron $\mathrm{x}$-ray experiment. However, it may be feasible to try $\mathrm{x}$-ray HBT experiments with brighter sources, such as those at the Advanced Light Source or the Advanced Photon Source.[11]

We would like to acknowledge useful discussions with.E. V. Shuryak, M. R. Howells, A. P. K. Wong, K. J. Randall, W. Yun, and E. Johnson. This work is supported by the U. S. Department of Energy Office of Basic Energy Sciences, Division of Material Science, under contract W-31-109-ENG-38. 


\section{'REFERENCES:}

[1]E. V. Shuryak, Sov. Phys. JETP 40, 30 (1975).

[2]M. R. Howells, "The X-Ray Hanbury Brown and Twiss Intensity Interferometer: A New Physics Experiment and A Diagnostic for Both X-Ray and Electron Beams at Light Source," ALS Technical Report, LSBL-27 (Feb. 1989).

[3]E. Gluskin, "Intensity Interferometry and its Application to Beam Diagnostics," in 1991 IEEE Particle Accel. Conf., (IEEE, New York, 1991), Vol. 2, p. 1169.

[4]E. Ikonen, Phys. Rev. Lett., 68, 2759 (1992).

[5]G. K. Shenoy, P. J. Viccaro, and D. M. Mills, "Characteristics of the 7-GeV Advanced Photon Source: A Guide for Users," Argonne National Laboratory Report, ANL-88-9 (1988); An ALS Handbook, Advanced Light Source, Berkeley (1989).

[6]M. Born and E. Wolf, Principles of Optics, (Pergamon, Oxford, 1980).

[7]E. Gluskin, I. McNulty, M. R. Howells, and P. J. Viccaro. Johnson, Nucl. Instr. Meth., A319, 226 (1992).

[8]E. Gluskin, I. McNulty, L. Yang, K. J. Randall, Z. Xu, and E. D. Johnson, Nucl. Instr. Meth., A347, 177 (1994).

[9]L. Yang, I. McNulty, and E. Gluskin, Rev. Sci. Instrum. 66, 2281 (1995).

${ }^{[10]}$ R. Hanbury Brown and R. Q. Twiss, Nature 177, 27 (1956); R. Hanbury Brown and R. Q. Twiss, Nature 178, 1046 (1956).

[11]Y. P. Feng, I. McNulty, and E. Gluskin, Argonne National Laboratory, unpublished information, 1994.

[12]J. W. Goodman, Statistical Optics, (Wiley, New York, 1985).

${ }^{[13] R . ~ L o u d o n, ~ T h e ~ Q u a n t u m ~ T h e o r y ~ o f ~ L i g h t, ~(C l e a r e n d o n, ~ O x f o r d, ~ 1983) . ~}$

${ }^{[14] R . ~ H a n b u r y ~ B r o w n ~ a n d ~ R . ~ Q . ~ T w i s s, ~ P r o c . ~ R o y a l ~ S o c . ~ A 243, ~} 291$ (1958). 


\section{FIGURE CAPTIONS:}

Fig. 1. (a). The pinhole geometry for either the HBT or the Young's experiment, where two apertures of area $A$ are completely encompassed by a single coherence area $A_{c}=L_{x c} L_{y c}$. (b) The slit geometry, where two apertures of area $A=L_{x} L_{y}$ cover $M_{x}=L_{x} / L_{x c}$ horizontally coherent modes, but less than one vertically coherent mode. Each slit can be divided into $M_{x}$ imaginary coherent subcells, with each subcell (shaded area) extending over just one horizontal coherence width $L_{x c}$.

Fig. 2. Calculation of the correlation signal and noise in a HBT experiment using the slit geometry. (a) The total correlation signal is the incoherent sum of the individual correlation signals arising from $M_{x}$ pairs of mutually coherent subcells as indicated by the double-arrowhead lines. (b) The total noise-squared is the sum of the individual noise-squared of $M_{x}^{2}$ possible combinations of subcells, regardless of whether they are located inside or outside of a single coherence area. One such possible combination is between the $i$ th subcell in the upper slit and the $j$ th subcell in the lower slit.

Fig. 3. An improved HBT experimental configuration using one-dimensional detectors. The size of each detector element matches the coherence width $L_{x c}$. The signals from $M_{x}$ pairs of elements are correlated independently and then averaged. 
(a) Pinhole configuration

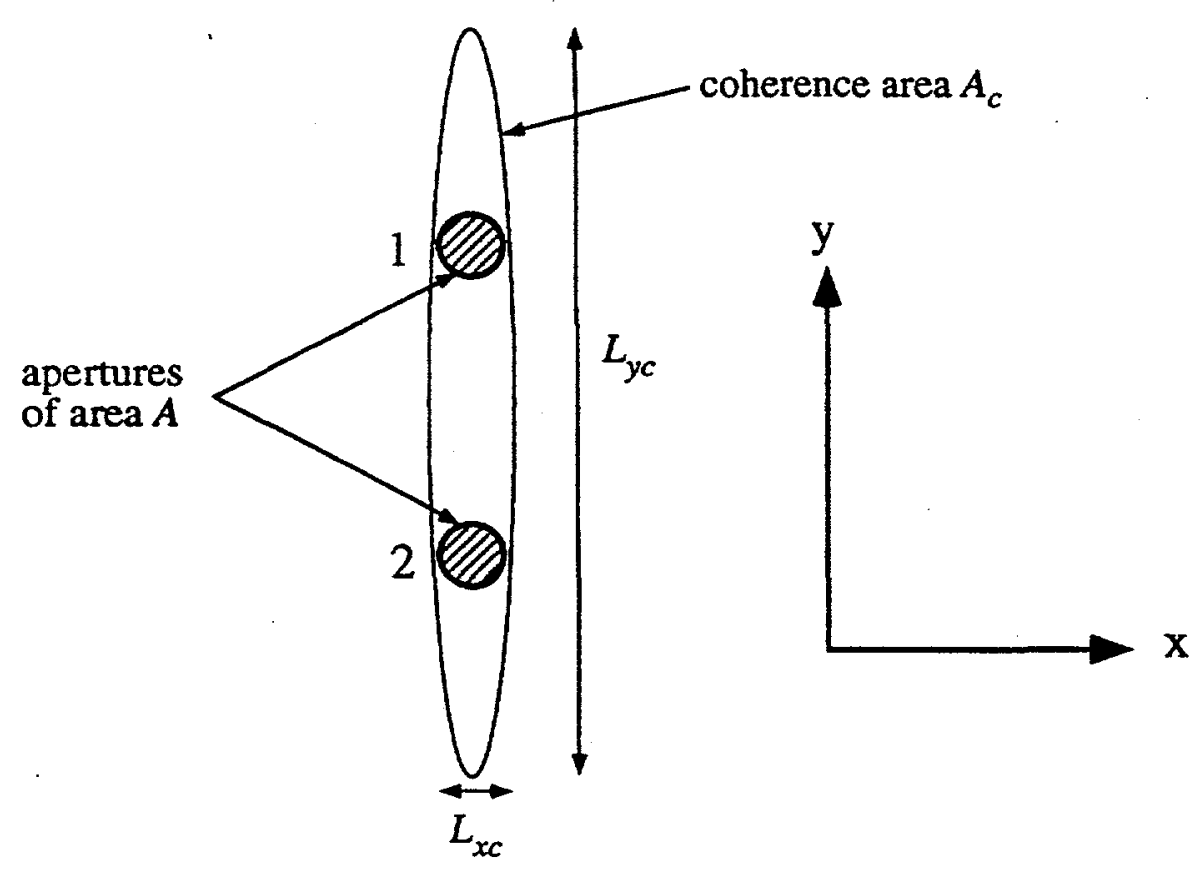

(b) Slit configuration

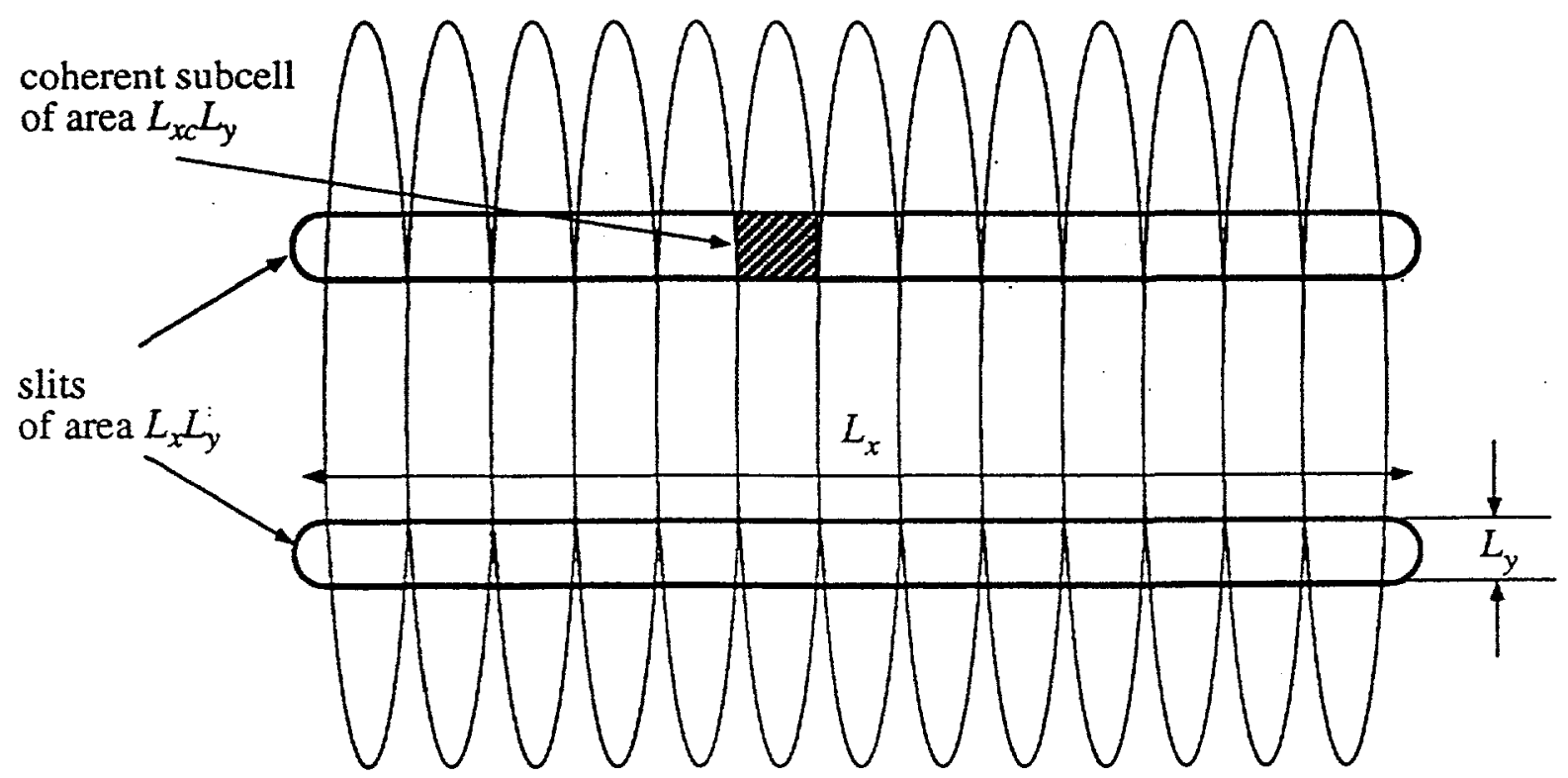

Fig. 1 
(a) correlator signal

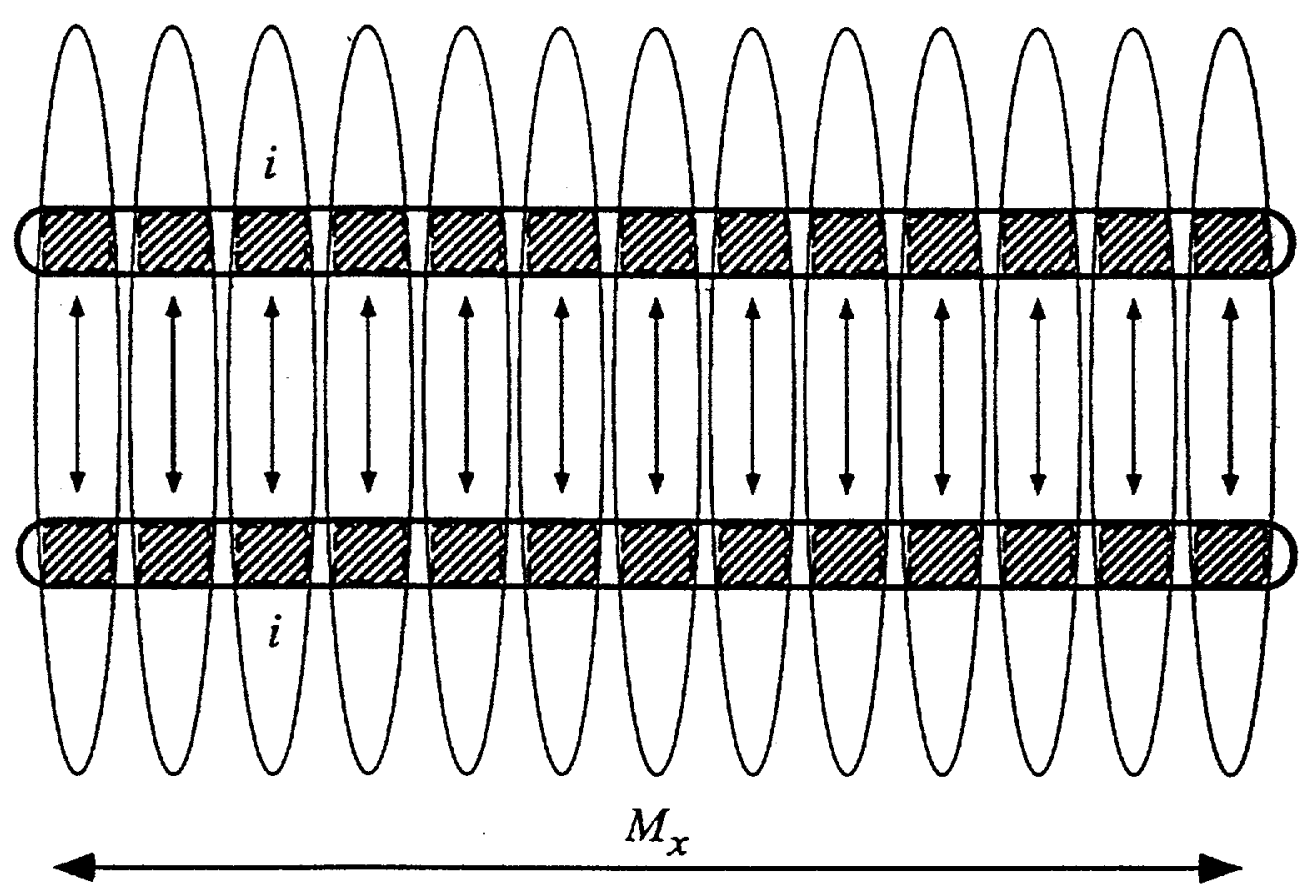

(b) correlator noise

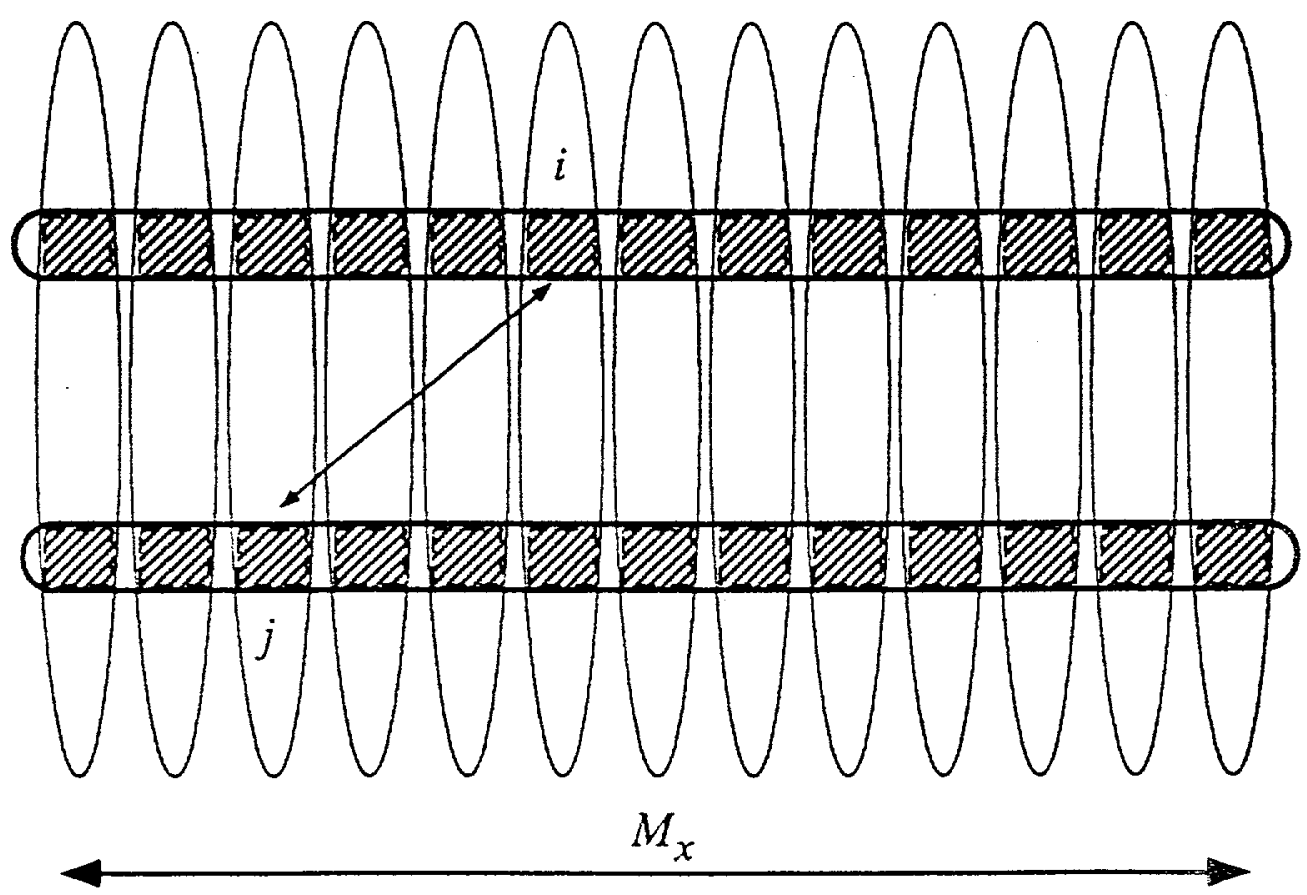

Fig. 2 


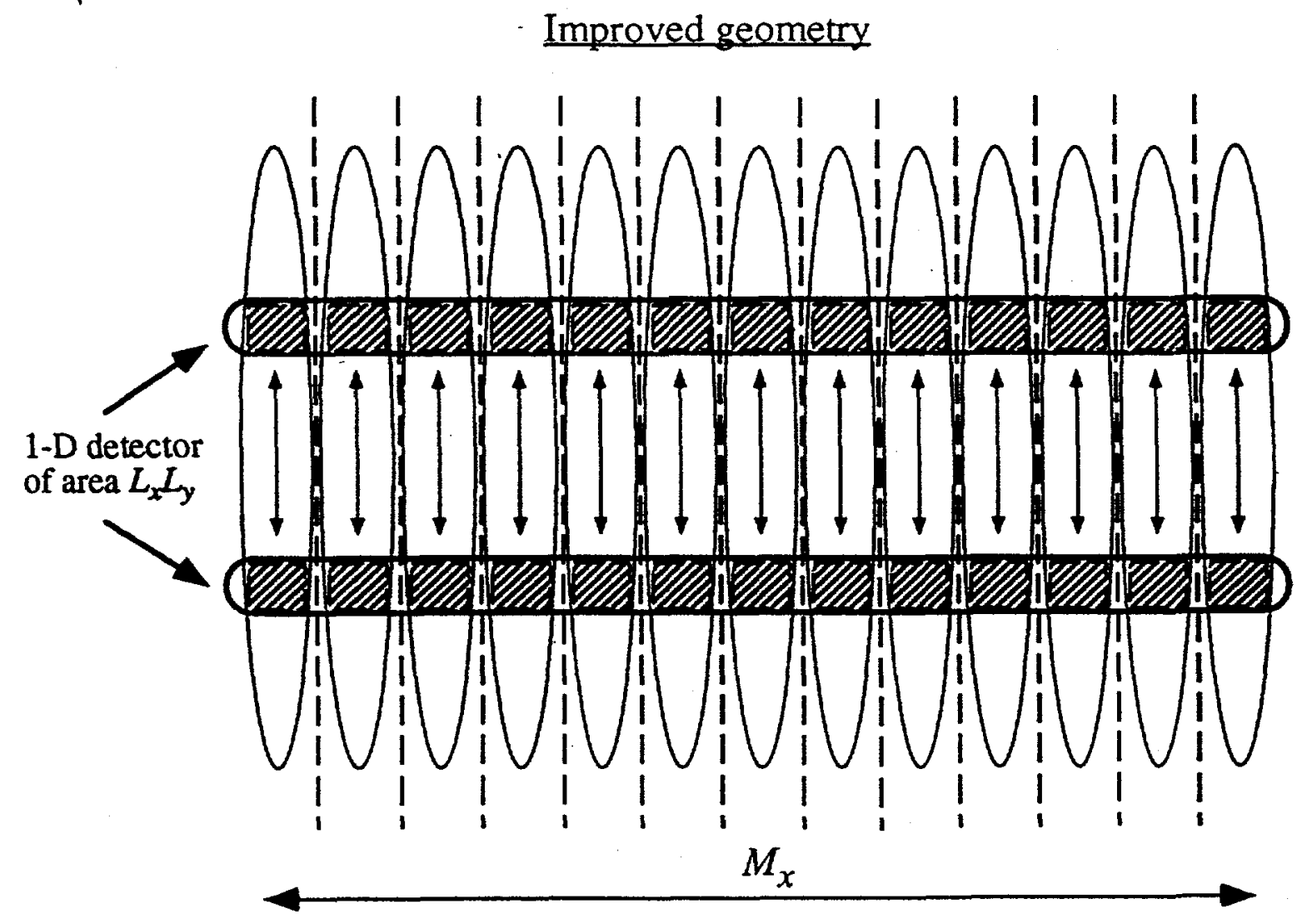

Fig. 3 